\title{
Relocking of intrinsic angular momenta in collisions of diatoms with ions: Capture
}

\section{of $\mathrm{H}_{2}(\mathrm{j}=0,1)$ by $\mathrm{H}_{2}^{+}$}

E. I. Dashevskaya, I. Litvin, E. E. Nikitin, and J. Troe

Citation: J. Chem. Phys. 145, 244315 (2016); doi: 10.1063/1.4972129

View online: http://dx.doi.org/10.1063/1.4972129

View Table of Contents: http://aip.scitation.org/toc/jcp/145/24

Published by the American Institute of Physics

\section{Articles you may be interested in}

Observation of enhanced rate coefficients in the $\mathrm{H} 2++\mathrm{H} 2 \rightarrow \mathrm{H} 3++\mathrm{H}$ reaction at low collision energies

J. Chem. Phys. 145, 244316244316 (2016); 10.1063/1.4972130

Hyperfine interactions and internal rotation in methanol

J. Chem. Phys. 145, 244301244301 (2016); 10.1063/1.4972004

Dissociative recombination of $\mathrm{HCl}_{+}, \mathrm{H} 2 \mathrm{Cl}+, \mathrm{DCl}$, and $\mathrm{D} 2 \mathrm{Cl}+$ in a flowing afterglow

J. Chem. Phys. 145, 244312244312 (2016); 10.1063/1.4972063

Coherent dynamics of V-type systems driven by time-dependent incoherent radiation

J. Chem. Phys. 145, 244313244313 (2016); 10.1063/1.4972140

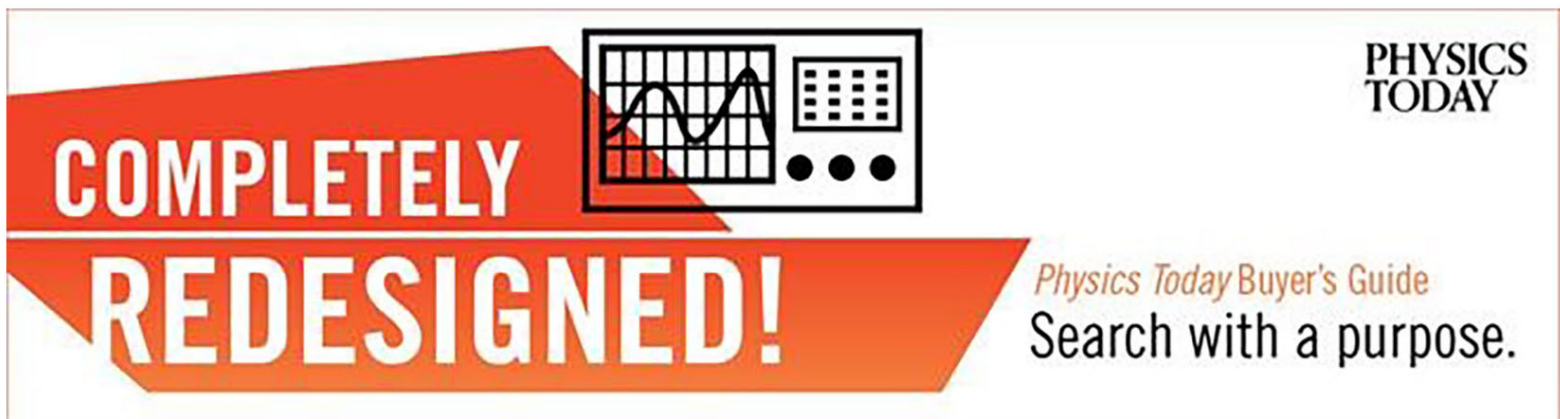




\title{
Relocking of intrinsic angular momenta in collisions of diatoms with ions: Capture of $\mathrm{H}_{2}(j=0,1)$ by $\mathrm{H}_{2}{ }^{+}$
}

\author{
E. I. Dashevskaya, ${ }^{1,2}$ I. Litvin, ${ }^{3}$ E. E. Nikitin, ${ }^{1,2}$ and J. Troe ${ }^{2,3, a)}$ \\ ${ }^{1}$ Schulich Faculty of Chemistry, Technion-Israel Institute of Technology, Haifa 32000, Israel \\ ${ }^{2}$ Max-Planck-Institut für Biophysikalische Chemie, Am Fassberg 11, D-37077 Göttingen, Germany \\ ${ }^{3}$ Institut für Physikalische Chemie, Universität Göttingen, Tammannstrasse 6, D-37077 Göttingen, Germany
}

(Received 25 October 2016; accepted 30 November 2016; published online 29 December 2016)

\begin{abstract}
Rate coefficients for capture of $\mathrm{H}_{2}(j=0,1)$ by $\mathrm{H}_{2}{ }^{+}$are calculated in perturbed rotor approximation, i.e., at collision energies considerably lower than Bhc (where B denotes the rotational constant of $\mathrm{H}_{2}$ ). The results are compared with the results from an axially nonadiabatic channel (ANC) approach, the latter providing a very good approximation from the low-temperature Bethe-Wigner to the high temperature Langevin limit. The classical ANC approximation performs satisfactorily at temperatures above $0.1 \mathrm{~K}$. At $0.1 \mathrm{~K}$, the rate coefficient for $j=1$ is about $25 \%$ higher than that for $j=0$ while the latter is close to the Langevin rate coefficient. The Bethe-Wigner limit of the rate coefficient for $j=1$ is about twice that for $j=0$. The analysis of the relocking of the intrinsic angular momentum of $\mathrm{H}_{2}$ during the course of the collision illustrates the significance of relocking in capture dynamics in general. Published by AIP Publishing. [http://dx.doi.org/10.1063/1.4972129]
\end{abstract}

\section{INTRODUCTION}

The capture of a diatom in a closed electronic and an adiabatically isolated vibrational state by an ion, after separating the center-of-mass $(\mathrm{CM})$ motion and taking into account the conservation of total angular momentum, is described by four variables: the CM distance radial coordinate $R$ of relative motion of the fragments, the absolute value $j$ of the intrinsic angular momentum $\mathbf{j}$ of the diatom, its angle of precession $\phi$ about $\mathbf{R}$, and its angle of nutation $\beta$ with respect to $\mathbf{R}$. The analysis of the dynamics can be simplified in the perturbed rotor approximation where the coupling between different $j$ states is ignored, and each $j$ state is handled independently under the assumption that the absolute value of $\mathbf{j}$ is conserved (this requires low collision energies, $E \ll \Delta E_{r o t}$ where $\Delta E_{r o t}$ denotes the energy for rotational excitation). The classical version of this approximation (with two coordinates $R, \phi$ and two conjugate momenta $P_{R}, P_{\phi}$, the latter being related to the nutation angle $\beta$ ) was elaborated in Refs. 1 and 2 for charge-induced dipole and charge-permanent quadrupole interactions. It was found that, in the course of the capture process, the projection of the intrinsic angular momentum onto the collision axis changes noticeably. This is an effect of nutation which, due to its coupling to the relative motion, occurs in a non-periodic manner and in the course of the collision noticeably changes the projection of $\mathbf{j}$ onto the collision axis $\mathbf{R}$.

The change of the rotational polarization of the diatom, i.e., the relocking of the projection of $\mathbf{j}$ onto the collision axis, results in the following properties of capture:

(i) The conventional adiabatic channel (AC) model, such as, e.g., described in Ref. 3, provides a fair approximation for $j$-specific cross sections of unpolarized diatoms, although this approach ignores radial and

a)Email: jtroe@mpibpc.mpg.de rotational (Coriolis) nonadiabatic effects. This is a consequence of the fact that, for large values of the total angular momentum (quantum number $J$ ), the locking (which causes transitions between different AC states) occurs at larger distances than the effective capture distances so that, on average, the neglect of Coriolis coupling does not affect the population of the initially unpolarized $\mathrm{AC}$ states in the region of the centrifugal barriers.

(ii) The $j$-specific capture cross sections for rotationally polarized diatoms show a marked dependence on the polarization state which is not accounted for in the AC model. Because of incomplete locking, the passage over the centrifugal barriers is accompanied by considerable gyroscopic effects. A similar effect is well known for atomic collisions. ${ }^{4}$

(iii) As discussed in Refs. 5-7, the relocking of the intrinsic angular momentum with respect to the collision axis can be taken into account within an axially nonadiabatic channel (ANC) approach which diagonalizes the Coriolis coupling at fixed interfragment distances. However, the incorporation of Coriolis effects into the ANC potentials generates new regions of radial nonadiabatic coupling (in comparison to those of an AC approach) which cannot be ignored and thus requires further investigation.

We expect that the results described in (i) and (iii) will be substantially modified when the condition $J \gg 1$ is not valid, such that the discrete character of angular momenta and the quantum character of the relative motion become important. Therefore, the aim of the present work is twofold. First, we study various decoupling phenomena at low energies where the condition of a perturbed rotor approximation is fulfilled. Second, we present accurate rate coefficients within a coupledstate approach. We consider the capture of $\mathrm{H}_{2}(j=0,1)$ by 
$\mathrm{H}_{2}{ }^{+}$as an example which represents one of the most promising systems for an experimental study of quantum effects in the capture (see, e.g., the recent Refs. 8-10). For this system, the condition of low collision energies takes the form $E<<6$ Bhc where $\mathrm{B}=60.85 \mathrm{~cm}^{-1}$ is the rotational constant of $\mathrm{H}_{2}$. Accordingly, the article is organized as follows. Section II describes a choice of basis sets for the description of capture in the perturbed rotor states $j=0$ and $j=1$, taking into account the charge-induced dipole and chargepermanent quadrupole interactions. It also provides a general description of the potentials, which are characterized by two dimensionless parameters. Section III considers the change of rotational polarization of the diatom in the capture event, i.e., the relocking. Section IV provides numerical results for the energy-dependent capture rate coefficients in the capture of $\mathrm{H}_{2}(j=0,1)$ by $\mathrm{H}_{2}{ }^{+}$. Section $\mathrm{V}$ concludes the article. A more detailed analysis of the energy dependence of the rate coefficients in terms of decoupling within the ANC approach is given in the Appendix. This analysis permits a deeper insight into capture dynamics in general.

\section{INTERACTION POTENTIALS, BASIS FUNCTIONS AND THE HAMILTONIANS}

The ion-diatom interaction energy is written as a sum of the isotropic and anisotropic ion-induced dipole and the ionquadrupole interactions

$V(R, \vartheta)=-\frac{q^{2} \alpha}{2 R^{4}}+\left(-\frac{q^{2} \Delta \alpha}{3 R^{4}}+\frac{q Q}{R^{3}}\right) \frac{\left(3 \cos ^{2} \vartheta-1\right)}{2}$,

where $q$ is the charge of the ion, $\alpha$ is the mean polarizability, $\alpha=\left(\alpha_{\|}+2 \alpha_{\perp}\right) / 3, \Delta \alpha=\alpha_{\|}-\alpha_{\perp}, Q$ is the quadrupole moment of the molecule, and $\vartheta$ is the angle between the molecular and the collision axis. The Hamiltonian $\hat{H}$ of the system is restricted to the sum of $V(R, \vartheta)$, the relative radial kinetic energy $\hat{P}_{R}^{2} / 2 \mu$, the relative rotational energy $\hat{\mathbf{I}}^{2} / 2 \mu R^{2}$, and the rotational energy of the diatom $E_{r o t}(j)$,

$$
\hat{H}=\hat{P}_{R}^{2} / 2 \mu+\hat{\mathbf{I}}^{2} / 2 \mu R^{2}+V(R, \vartheta)+E_{r o t}(j) .
$$

Here $\hat{P}_{R}$ is the radial momentum of relative motion, $\mu$ is the reduced mass of the collision partners, and $\hat{\mathbf{I}}^{2}$ can be expressed through the total $(\mathbf{J})$ and intrinsic (j) angular momenta as $\hat{\mathbf{l}}^{2}=(\hat{\mathbf{J}}-\hat{\mathbf{j}})^{2}$. In the following, we use the scaled variables for the distance $R, \rho=R / R_{L}$, for the wave vector $k$, $\kappa=k R_{L}$, and for the energy $E, \varepsilon=E / E_{L}\left(\right.$ where $R_{L}=\sqrt{\mu q^{2} \alpha} / \hbar$ and $\left.E_{L}=\hbar^{2} / \mu R_{L}^{2}\right)$; the scaled parameters of the interaction potential are $a=2 \Delta \alpha / 15 \alpha, b=2 \mu q Q / 5 R_{L} \hbar^{2}$. We note in passing that the Langevin rate coefficient (for $a=b=0$ ) $k_{\mathrm{L}}=2 \pi \sqrt{q^{2} \alpha / \mu}$ is related to $R_{L}$ by $k_{\mathrm{L}}=2 \pi \hbar R_{\mathrm{L}} / \mu$.

The classical perturbed rotor ( $\mathrm{PRCl}$ ) Hamiltonian, $H^{\mathrm{PRCl}}$, is obtained from Eq. (2.2) by averaging $H$ over the angle of proper rotation of the diatom in a state with fixed $j .{ }^{1,2}$ Then, $H^{\mathrm{PRCl}}$ depends on the conjugate dynamical variables $\left(R, P_{R}\right)$ and $\left(\varphi, p_{\varphi}\right)$ where $\varphi$ denotes the precession angle of the vector $\mathbf{j}$ about the collision axis and $p_{\varphi}$ is the projection of $\mathbf{j}$ onto the collision axis. In addition, $H^{\mathrm{PRCl}}$ depends parametrically on the total and intrinsic angular momenta $J$ and $j$.
The quantum perturbed rotor (PRQ) Hamiltonian $\hat{H}^{\mathrm{PRQ}}$ is obtained by projecting $\hat{H}$ onto the manifold of states $|J, M, j, \gamma\rangle$ each labeled by the quantum numbers $J, M, j$ (corresponding to the total angular momentum, its Z-projection in a space-fixed (SF) frame and the intrinsic angular momentum of the diatom) as well as by an additional quantum number $\gamma$ that defines the choice of the basis. The wave functions $|J, M, j, \gamma\rangle$ depend on the set of angular variables $\Xi$ that describe the orientation of the collision axis in a SF frame and the orientation of the rotor axis either in the SF or in the body-fixed (BF) frame. In addition, these functions may also depend on $\rho$. The Hamiltonian $\hat{H}^{\text {PRQ }}$ describes the radial motion of the partners and it depends on the quantum number $\gamma$ of a chosen representation. In scaled variables and using Eq. (2.2) this Hamiltonian is represented by a matrix $\hat{\eta}$ with the elements $\eta_{\gamma \gamma^{\prime}}^{J j}$ in the form

$$
\eta_{\gamma \gamma^{\prime}}^{J j}=-\delta_{\gamma \gamma^{\prime}} d^{2} / 2 d \rho^{2}+v_{\gamma \gamma^{\prime}}^{J j}(\rho) .
$$

Here, the first term of the r.h.s. represents the diagonal radial kinetic energy operator and the second term results from the effective interaction potential and the interchannel coupling. In what follows, we refer to four different bases: basis of free states (FS), standard adiabatic channel (AC) basis, parityadopted AC basis PAAC, and the axially nonadiabatic (ANC) basis. The respective quantum numbers $\gamma$ for these bases are: relative angular momentum $\ell$ (i.e., $\gamma \equiv \ell$ for FS with the $\rho$-independent functions $|J, M, j, \ell ; \mathrm{FS}\rangle)$, projection of $\mathrm{j}$ onto the collision axis $\omega$ for $\mathrm{AC}$ (i.e., $\gamma \equiv \omega$ with the $\rho$-independent functions $|J, M, j, \omega ; \mathrm{AC}\rangle)$, absolute value of the projection of $\mathbf{j}$ onto the collision axis $|\omega|$ labeled by the parity quantum numbers \pm for PAAC (i.e., $\gamma=|\omega|^{ \pm}$with the $\rho$-independent functions $\left.\left.\left.|J, M, j,| \omega\right|^{ \pm} ; \mathrm{ANC}\right\rangle\right)$, and the quantum number $s$ for the ANC, asymptotically related to the FS quantum number $\ell$ (i.e., $\gamma=s, s=J-\ell$ with the $\rho$-dependent functions $|J, M, j, s ; \mathrm{ANC}\rangle)$ by the relation $\left.|J, M, j, s ; \mathrm{ANC}\rangle\right|_{\rho \rightarrow \infty} \rightarrow$ $|J, M, j, \ell ; \mathrm{FS}\rangle$. The off-diagonal matrix elements in Eq. (2.3) originate from the anisotropic interaction (in the FS basis), the Coriolis interaction (in the AC basis), the reduced Coriolis interaction (in the PAAC basis), and the radial nonadiabatic interaction (in the ANC basis).

Of course, the AC, PAAC, and ANC functions can be expressed through the FS functions. For instance, the FS-AC relation reads

$|J, M, j, \ell ; \mathrm{FS}\rangle=\sum_{\omega}(-1)^{j-\omega} C_{J \omega ; j-\omega}^{\ell 0}|J, M, j, \omega ; \mathrm{AC}\rangle$,

where $C_{J \omega ; j-\omega}^{\ell 0}$ stand for the Clebsch-Gordan coefficients. Here the FS states are given by a standard Clebsch-Gordan contraction of two linear rotor functions in the SF frame, (one for the collision axis and another for the diatom and $\mathrm{AC}$ states). The AC states are expressed through the symmetric top (ST) functions (with vanishing third angle) in the SF frame and the linear rotor function in the BF frame (the former for the collision axis, and the latter for the diatom).

Passing now to the cases $j=0$ and $j=1$, we note that, for $j=0$, the matrix $v_{\gamma \gamma^{\prime}}^{J j}$ consists of a single element with $J=\ell$,

$$
\left.v_{\gamma \gamma^{\prime}}^{J j=0}\right|_{J=\ell}=\ell(\ell+1) / 2 \rho^{2}-1 / 2 \rho^{4} \text {. }
$$


For $j=1$, the expression for $v_{\gamma \gamma^{\prime}}^{J j}$ depends on the representation. In the following, we suppress the quantum numbers $M$ (which does not enter into the Hamiltonian $\hat{\eta}$ ) and $j$ (which is fixed to $j=1$ ) and consider the AC, PAAC, and ANC representations:

In the $\mathrm{AC}$ basis $|J, \omega ; \mathrm{AC}\rangle$, the quantum numbers are $\omega=-1,0,1$ and the matrix $\left.v_{\gamma \gamma^{\prime}}^{J, j=1}\right|_{\mathrm{AC}} \equiv{ }^{\mathrm{AC}} v_{\omega \omega^{\prime}}^{J}$ assumes the form

$$
\begin{aligned}
\mathrm{AC}_{v_{\omega \omega}^{J, j=1}}^{J, j} & \frac{J(J+1)+2-2 \omega^{2}}{2 \rho^{2}}-\frac{1}{2 \rho^{4}} \\
& +\frac{2-3 \omega^{2}}{2}\left(\frac{a}{\rho^{4}}+\frac{b}{\rho^{3}}\right), \omega=-1,0,1, \\
\mathrm{AC}_{v_{0, \pm 1}^{J, j=1}=} & i \sqrt{J(J+1)} / \sqrt{2} \rho^{2} .
\end{aligned}
$$

In the PAAC basis $\left.|J,| \omega\right|^{ \pm}$; PAAC $\rangle$of definite parity $P$, the quantum numbers $|\omega|^{ \pm}=0^{+}, 1^{+}, 1^{-}$correspond to linear combinations of $\mathrm{AC}$ functions given by

$$
\begin{aligned}
\left|J, 1^{ \pm} ; \mathrm{PAAC}\right\rangle & =(1 / \sqrt{2})(|J, 1 ; \mathrm{AC}\rangle \mp|J,-1 ; \mathrm{AC}\rangle), \\
P & = \pm(-1)^{J}, \\
\left.\left.\mid J, 0^{+} ; \mathrm{PAAC}\right)\right\rangle & =|J, 0 ; \mathrm{AC}\rangle, \quad P=(-1)^{J} .
\end{aligned}
$$

The matrix $\left.v_{\gamma \gamma^{\prime}}^{J, j=1}\right|_{\mathrm{PAAC}} \equiv{ }^{\mathrm{PAAC}} v_{\gamma \gamma^{\prime}}^{J, j=1}$ here reads

$$
\begin{aligned}
& \operatorname{PAAC} v_{|\omega|^{ \pm},|\omega|^{ \pm}}^{J, j=1}={ }^{\mathrm{AC}} v_{\omega, \omega}^{J, j=1}, \\
& { }^{\operatorname{PAAC}} v_{0^{+}, 1^{+}}^{J 1}=i \sqrt{J(J+1)} / \rho^{2} .
\end{aligned}
$$

In the ANC basis $|J, s ; \mathrm{ANC}\rangle$, the quantum numbers $s=-1,0,1$ correspond to $(\rho, J)$-dependent linear combinations of PAAC functions given by

$|J, s ; \mathrm{ANC}\rangle=\left\{\begin{array}{c}\left.\left.\sum_{|\omega|^{ \pm}=0^{+}, 1^{+}} R_{s,|\omega|^{ \pm}}(\Theta)|J,| \omega\right|^{ \pm} ; \mathrm{PAAC}\right\rangle, \quad s=-1,1, \\ \left.\left.|J,| \omega\right|^{ \pm} ; \mathrm{PAAC}\right\rangle\left.\right|_{|\omega|^{ \pm}=1^{-}}, s=0 .\end{array}\right.$

Here $R(\Theta)$ is a standard $2 \times 2$ rotation matrix with the rotational angle $\Theta=\Theta(J, \rho)$,

$$
\Theta(J, \rho)=(1 / 2) \arctan \left(\frac{2 \sqrt{J(J+1)}}{1+3 b / 2 \rho+3 a / 2 \rho^{2}}\right) .
$$

The matrix $\left.v_{\gamma \gamma^{\prime}}^{J, j=1}\right|_{\mathrm{ANC}} \equiv{ }^{\mathrm{ANC}} v_{s s^{\prime}}^{J, j=1}$ reads

$$
{ }^{\mathrm{ANC}} v_{s s^{\prime}}^{J, j=1}={ }^{\mathrm{ANCA}} v_{s s^{\prime}}^{J, j=1}+{ }^{\mathrm{ANC}} \Delta v_{s s^{\prime}}^{J, j=1},
$$

where

$$
\begin{aligned}
{ }^{\mathrm{ANCA}} v_{s s}^{J, j=1}= & \frac{J(J+1)+s^{2}}{2 \rho^{2}}-\frac{1}{2 \rho^{4}}-\left(\frac{b}{\rho^{3}}+\frac{a}{\rho^{4}}\right) \frac{2-3 s^{2}}{4} \\
& +\frac{s}{2} \sqrt{\left(\frac{3 a}{2 \rho^{4}}+\frac{3 b}{2 \rho^{3}}+\frac{1}{\rho^{2}}\right)^{2}+\frac{4 J(J+1)}{\rho^{4}}}
\end{aligned}
$$

$$
{ }^{\mathrm{ANC}} \Delta v_{s s}^{J, j=1}=\frac{s^{2}}{2}\left(\frac{\partial \Theta}{\partial \rho}\right)^{2}
$$

and

$$
{ }^{\mathrm{ANC}} v_{1,-1}^{J, j=1}=-\frac{\partial \Theta}{\partial \rho} \frac{d}{d \rho}+\frac{1}{2} \frac{\partial^{2} \Theta}{\partial \rho^{2}}
$$

Here ${ }^{\mathrm{ANCA}} v_{s s}^{J, j=1}$ results from the interaction potential and the relative rotation, while ${ }^{\mathrm{ANC}} \Delta v_{s s}^{J, j=1}$ results from the diagonal correction of the radial nonadiabatic coupling. Note that ${ }^{\mathrm{ANC}} v_{s s}^{J, j=1}$ differs from the ANC potentials introduced in Refs. 6 and 7, since the latter do not include the diagonal nonadiabatic correction and are identical to our ANCA potentials. Due to the centrifugal repulsion, all ANC and ANCA potentials (except those with $J=1, s=-1$ ) exhibit an asymptotical $\rho^{-2}$ dependence. Superimposed on this are $\rho^{-3}$ - (chargequadrupole interaction) and $\rho^{-4}$-dependences (charge-induced dipole interaction and diagonal nonadiabatic corrections). For the $J=1, s=-1$ state, only the former survives such that asymptotically, at $\rho \gg 3|b| /(2 J+1)^{2}$, the ANC and ANCA potentials read

$$
\begin{aligned}
\left.{ }^{\mathrm{ANCA}} v_{s s}^{J, j=1}\right|_{J=1, s=-1} & =-\frac{1}{2 \rho^{4}}\left(1+b^{2} / 3\right), \\
\left.{ }^{\mathrm{ANC}} v_{s s}^{J, j=1}\right|_{J=1, s=-1} & =-\frac{1}{2 \rho^{4}}\left(1+5 b^{2} / 18\right) .
\end{aligned}
$$

The correction in the parentheses of the ANCA potential can be calculated in second order with respect to the chargequadrupole interaction in the FS basis, while this correction to the ANC potential arises from the second order chargequadrupole interaction, being modified by the diagonal nonadiabatic corrections. Note that the matrix $\hat{\eta}$ in the AC basis contains four off-diagonal elements (from Coriolis coupling); in the PAAC basis there are two off-diagonal elements (from Coriolis coupling) and in the ANC basis there are two offdiagonal elements (from the radial coupling). In the two limits $\rho \rightarrow 0$ and $\rho \rightarrow \infty$, the ANC states coincide with the PAAC states and the FS states. This correlation is summarized in Table I.

The wave functions in the asymptotic regions are most clearly visualized in the $J$-helicity representation for $J \gg 1$ (with $\ell=J-j_{J}$, see Ref. 11) which implies a passage to the semiclassical approximation. With the classical vector $\mathbf{J}$

\begin{tabular}{|c|c|c|c|}
\hline $\begin{array}{l}\mid J, s ; \text { ANC }\rangle\left.\right|_{\rho \rightarrow 0} \\
\left.=\left.|J,| \omega\right|^{ \pm} ; \text {PAAC }\right\rangle\end{array}$ & $|J, s ; \mathrm{ANC}\rangle$ & $\begin{array}{l}\left.|J, s ; \mathrm{ANC}\rangle\right|_{\rho \rightarrow \infty} \\
\quad=|J, \ell ; \mathrm{FS}\rangle\end{array}$ & Parity $P$ \\
\hline$|\omega|^{ \pm}=1^{+}$ & $s=-1$ & $\ell=J-1$ & $P=(-1)^{J}$ \\
\hline$|\omega|^{ \pm}=1^{-}$ & $s=0$ & $\ell=J$ & $P=-(-1)^{J}$ \\
\hline$|\omega|^{ \pm}=0^{+}$ & $s=1$ & $\ell=J+1$ & $P=(-1)^{J}$ \\
\hline
\end{tabular}
taken as the polar axis, and the vector $\rho$ taken as the zero line for the azimuth of the rotor, the rotor wave functions in polar coordinates $\vartheta_{J}, \varphi_{\rho}$ in the BF frame are given in Table II.

We finally note that the $\rho$-dependence of the rotor functions $\mid J, s$; ANC $\rangle$ implies a change of the locking (i.e., a relocking) of $\mathbf{j}$ with respect to $\boldsymbol{\rho}$. The relocking decreases the effective potentials in the set of channels with $s=-1$. An example of this decrease is given in Eq. (2.12) where the potential is purely attractive notwithstanding the nonzero value of the total angular momentum $J=1$. This lowering of the potentials has the following consequence. At low energies, the main contribution to the capture comes from those channels of the set $s=-1$ for which the motion is classically allowed. This motion

TABLE I. Adiabatic correlation of ANC states with PAAC and FS states for $j=1$. 
TABLE II. Asymptotic rotor functions in the $J$-helicity representation for $b>0$ in polar coordinates $\vartheta_{J}, \phi_{\rho}((\mathrm{X})$ means degenerate states).

\begin{tabular}{|c|c|c|c|}
\hline $\begin{array}{l}\text { Non-normalized rotor } \\
\text { functions at } \rho \rightarrow 0\end{array}$ & $|J, s ; \mathrm{ANC}\rangle$ & $\begin{array}{l}\text { Non-normalized rotor } \\
\text { functions at } \rho \rightarrow \infty\end{array}$ & $\begin{array}{l}\left.|J, s ; \mathrm{ANC}\rangle\right|_{\rho \rightarrow \infty} \\
\quad=|J, \ell ; \mathrm{FS}\rangle\end{array}$ \\
\hline $\sin \vartheta \sin \varphi(X)$ & $s=-1$ & $\sin \vartheta_{J} \exp \left(i \phi_{\rho}\right)$ & $\ell=J-1$ \\
\hline $\cos \vartheta(\mathrm{X})$ & $s=0$ & $\cos \vartheta_{J} \quad$ (X) & $\ell=J$ \\
\hline $\sin \vartheta \cos \varphi$ & $s=1$ & $\sin \vartheta_{J} \exp \left(-i \phi_{\rho}\right)$ & $\ell=J+1$ \\
\hline
\end{tabular}

is coupled to the classically forbidden motion in the set of channels $s=1$. Since the latter do not contribute substantially to the capture, one can neglect the coupling as given by Eq. (2.11) and describe the capture dynamics in the decoupling approximation. This approximation is discussed in detail in the Appendix. It is also used in Section III for a description of relocking.

\section{ROTATIONAL POLARIZATION OF THE DIATOM IN THE CAPTURE EVENT}

The locking of the intrinsic angular momentum to the collision axis is described by the $\rho$-dependent matrix of a polarization operator in the chosen basis of the rotational functions (AC, PAAC, or ANC). The polarization matrix, together with the wave function as represented in the form of an expansion in rotational functions, allows one to determine the evolution of the expectation value of a locking feature as a function of the distance $\rho$ between the collision partners only. A limited information on the change of the locking (i.e., relocking) along $\rho$ is provided by the diagonal elements of the polarization matrix. But this information is sufficient for the description of relocking in the decoupling approximation which is used for the general analysis of the energy dependence of capture cross sections in the Appendix.

Since a definite orientation of $\mathbf{j}$ with respect to the collision axis is not compatible with the conservation of the total parity (see above), we characterize the polarization by an alignment index that is defined as the expectation value $\langle\hat{A}\rangle$ of the $\mathbf{R}$-component of the alignment operator $\hat{A}=2-3 \hat{j}_{R}^{2}$ (being proportional to the expectation value of the charge-quadrupole interaction). Since relocking is due to the interplay of electrostatic and Coriolis interactions and since the latter is ignored in the AC and PAAC basis functions, neither of them can be used for a description of the relocking. Therefore, the expectation values of $A$ do not depend on $\rho$ and are given by

$$
\begin{aligned}
\langle J, M, \omega|\hat{A}| J, M, \omega\rangle & =\left\langle J, M,|\omega|^{p}|\hat{A}| J, M,|\omega|^{p}\right\rangle \\
& =\left\{\begin{array}{c}
2 \text { for } \omega=0, \\
-1 \text { for } \omega= \pm 1 .
\end{array}\right.
\end{aligned}
$$

On the other hand, the three ANC alignment indices are

$$
\begin{aligned}
A_{-1}(J, \rho) & \equiv\langle J, M, s|\hat{A}| J, M, s\rangle_{s=-1}=3 \sin ^{2} \Theta-1, \\
A_{0}(J, \rho) & \equiv\langle J, M, s|\hat{A}| J, M, s\rangle_{s=0}=-1, \\
A_{1}(J, \rho) & \equiv\langle J, M, s|\hat{A}| J, M, s\rangle_{s=1}=3 \cos ^{2} \Theta-1,
\end{aligned}
$$

where $\Theta \equiv \Theta(J, \rho)$ is defined by Eq. (2.10).
The limiting, $\mathrm{AC}$ and $\mathrm{FS}$, expressions, $A_{s}^{\mathrm{AC}}(J)$ $=\lim _{\rho \rightarrow 0} A_{s}(J, \rho)$ and $A_{s}^{\mathrm{FS}}(J)=\lim _{\rho \rightarrow \infty} A_{s}(J, \rho)$ respectively, are

$$
\begin{aligned}
& A_{-1}^{\mathrm{AC}}=-1 ; \quad A_{-1}^{\mathrm{FS}}=(J-1) /(2 J+1), \\
& A_{0}^{\mathrm{AC}}=-1 ; \quad A_{0}^{\mathrm{FS}}=-1, \\
& A_{1}^{\mathrm{AC}}=2 ; \quad A_{-1}^{\mathrm{FS}}=(J+2) /(2 J+1) .
\end{aligned}
$$

Nonvanishing asymptotic alignment $A_{s}^{\mathrm{FS}}($ at $\rho \rightarrow \infty)$ is termed as "kinematic polarization," 12 and it arises from the restrictions imposed by the conservation of total angular momentum and the normal orientation of the relative angular momentum with respect to the collision axis. This kind of polarization was extensively discussed in Ref. 13. Complementary to it, one can also understand the dynamic polarization that arises from the interplay of the anisotropic electrostatic and the Coriolis interaction at finite values of $\rho$, i.e., from the locking. ${ }^{14}$ For instance, the transformation of the rotor functions with decreasing $\rho$ for $s=1, s=-1$ from the limit of kinematic polarization to the AC limit of tight locking (see Table II) is the quantum counterpart of the precession/nutation motion of the rotor in the classical picture of capture.

We characterize the change of the alignment resulting from relocking, by a relocking index $r_{s}(J, \rho)$, defined as

$$
r_{s}(J, \rho)=s \frac{A_{s}-A_{s}^{\mathrm{FS}}}{A_{s}^{\mathrm{AC}}-A_{s}^{\mathrm{FS}}} .
$$

For $s=0$, Eq. (3.4) yields $r_{0}(J, \rho)=0$. This accounts for the fact that, with the potential ${ }^{\mathrm{ANC}} v_{0}^{J, j=1}(\rho)$, the alignment index does not depend on $\rho$, and the vanishing value of $r_{0}(J, \rho)$ implies that the capture in this potential is not associated with a change of the locking. For $s= \pm 1$ and fixed values of $J$, the functions $r_{ \pm 1}(J, \rho)$ characterize the extent of locking as a function of the distance. If the term with the parameter $a$ in Eq. (2.9) can be neglected (which is often the case), the function $r_{s}(J, \rho)$ shows a universal behavior when expressed in terms of the scaled variable $\rho / b$. If capture is associated to the attaining of a certain distance $\rho_{J, s}^{j=1}$, then the locking index $r_{s}\left(J, \rho_{J, s}^{j=1}\right)$ can be considered as a characteristic polarization property in the capture event. Fig. 1 shows the plots of $r_{s}(J, \rho)$ vs $\rho$ for various values of $J$, taking the capture of $\mathrm{H}_{2}(j=1)$ by $\mathrm{H}_{2}{ }^{+}$ as an example. The symbols here mark the distances $\rho_{J, s}^{j=1}$ at which the effective potentials assume their maximum values. These distances therefore denote effective capture distances, see below.

Two features deserve special notice. First, the lowest curve $(J=1, s=-1)$ is not associated with a well-defined capture distance, there is thus no symbol on this curve. Second, the state $(J=0, s=1)$ is not associated with a change of polarization, 


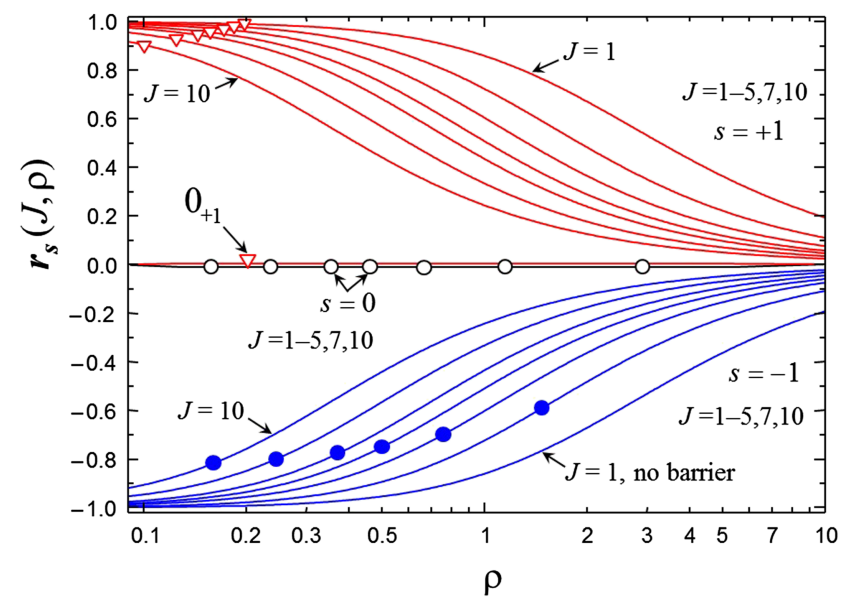

FIG. 1. Dependence of the relocking index $r_{s}$ of Eq. (3.4) on the scaled distance $\rho$ between the collision partners for different ${ }^{A N C} v_{s}^{J, j=1}$ potentials. The symbols refer to the capture distances $\rho_{J_{S}}^{j}$ (see text; filled circles, open circles, and triangles for $s=-1,0,1$; the horizontal line $r_{s}=0$ corresponds to $r_{1}(0, \rho)$ and $r_{0}(J, \rho)$; calculations are for capture of $\mathrm{H}_{2}(j=1)$ by $\mathrm{H}_{2}{ }^{+}$).

since for this case $\ell$ becomes an exact quantum number, and the intrinsic momentum has a well-defined projection onto the vector $\rho$. Therefore, the alignment index $r_{1}(0, \rho)$ is represented by a horizontal line superimposed onto the set of horizontal lines for $r_{0}(J, \rho)$.

\section{CAPTURE RATE COEFFICIENTS}

The capture problem with the PR Hamiltonian can be solved in any of the above representations by integrating the coupled equations with incoming boundary conditions for each state and outgoing boundary conditions for all other states at large $\rho$ and for incoming boundary conditions toward the capture center at small $\rho$. In the latter case, the boundary conditions are specified by representing the solution of capture equations by a superposition of analytical WKB waves that travel to the capture center. With the Hamiltonian matrix $\eta_{\gamma \gamma^{\prime}}^{J j}$ in the basis $|J, j, \gamma ; \rho, \Xi\rangle$ and the boundary conditions specified by a set of quantum numbers $\Gamma$ at $\rho \rightarrow \infty$, this solution leads to a wave function of the form

$$
\Psi_{\Gamma}^{J j}(\rho, \Xi)=\sum_{\gamma} X_{\gamma ; \Gamma}^{J j}(\rho)|J, j, \gamma ; \rho, \Xi\rangle .
$$

The radial functions $X_{\gamma ; \Gamma}^{J j}(\rho)$ are calculated from a set of coupled equations

$$
\sum_{\gamma^{\prime}} \eta_{\gamma \gamma^{\prime}}^{J j} \mathrm{X}_{\gamma ; \Gamma}^{J j}=E \mathrm{X}_{\gamma ; \Gamma}^{J_{j}}
$$

The wave functions $\Psi_{\Gamma}^{J j}(\rho, \Xi)$ and $X_{\gamma ; \Gamma}^{J j}(\rho)$ in Eqs. (4.1) and (4.2), in addition to $J, j, \gamma$, are also parameterized by an asymptotic channel quantum number $\Gamma$ that specifies the boundary conditions. The asymptotic behavior of the wave functions is described by a non-unitary scattering matrix $S_{\Gamma^{\prime}, \Gamma}^{J j}$ which, in turn, defines the capture probability $P_{\Gamma}^{J j}$ in the channel $\Gamma$ as

$$
P_{\Gamma}^{J j}=P_{J, \Gamma}^{j}=1-\sum_{\Gamma^{\prime}}\left|S_{\Gamma^{\prime}, \Gamma}^{J j}\right|^{2}
$$

TABLE III. Qualitative character of capture rate coefficients for $j=1$ in terms of scaled wave vectors $\kappa$ and scaled quadrupole moment $b$ (small anisotropic polarizability ignored).

\begin{tabular}{ll}
\hline \hline$\kappa, b$ relation & \multicolumn{1}{c}{ Characteristic features of the capture } \\
\hline$\kappa \rightarrow 0$ & Bethe-Wigner limit of the rate coefficient, $\chi^{j=1} \rightarrow \chi_{\mathrm{BW}}^{j=1}$ \\
$\sqrt{2 \kappa}<b$ & Prevailing effect of charge-quadrupole interaction \\
$\sqrt{2 \kappa}>b$ & Prevailing effect of charge-induced dipole \\
& interaction, $\chi^{j=1} \rightarrow \chi_{\mathrm{L}}=1$ \\
$\sqrt{2 \kappa} \gg 1$ & WKB character of capture \\
\hline \hline
\end{tabular}

For $j=0$, the asymptotic quantum number corresponds to the conserved quantum number $\ell=J$, and thus $\Gamma$ is redundant. For $j=1$, the asymptotic quantum numbers $\Gamma$ of different channels correspond either to the k-helicity quantum number $\Omega$ (the counterpart of $\omega$ in the AC basis at $\rho \rightarrow \infty$ ), or to the k-helicity quantum number $|\Omega|^{ \pm}$(the counterpart of $|\omega|^{ \pm}$in the PAAC basis at $\rho \rightarrow \infty$ ), or to the quantum number $S$ (the counterpart to $s$ in the ANC basis at $\rho \rightarrow \infty$, i.e., $S=-1,0,1$ for $\ell=J-1, J, J+1)$.

The capture probabilities $P_{\Gamma}^{J j}$ define the rate coefficients $k^{j}$ for capture of rotationally unpolarized rotors. Written in reduced form as $\chi^{j}=k^{j} / k_{\mathrm{L}}$ with the energy-independent Langevin rate coefficient $k_{\mathrm{L}}=2 \pi \sqrt{q^{2} \alpha / \mu}$ (which, for $\mathrm{H}_{2}+$ $\mathrm{H}_{2}{ }^{+}$capture, is equal to $2.10 \cdot 10^{-9} \mathrm{~cm}^{3} / \mathrm{s}$ or 0.342 a.u.), they read

$$
\begin{aligned}
& \chi^{j=0}(\kappa)=\frac{1}{2 \kappa} \sum_{\ell}(2 \ell+1) P^{\ell, j=0}(\kappa), \\
& \chi^{j=1}(\kappa)=\frac{1}{6 \kappa} \sum_{J, \Gamma}(2 J+1) P_{\Gamma}^{J, j=1}(\kappa),
\end{aligned}
$$

where the scaled wave vector $\kappa$ is defined in Sec. II. The highenergy limits of $\chi^{j=0}(\kappa)$ and $\chi^{j=1}(\kappa)$ are the Langevin rate coefficient, i.e., $\chi_{\mathrm{L}}=1$. The zero-energy value of $\chi^{j=0}(\kappa)$ is the Bethe-Wigner rate coefficient ${ }^{15,16}$ calculated for the charge-induced dipole interaction by Vogt and Wannier, ${ }^{17}$ $\left.\chi^{j=0}(\kappa)\right|_{\kappa \rightarrow 0} \equiv \chi_{\mathrm{BW}}^{j=0}=2$. The zero-energy value of $\chi^{j=1}(\kappa)$ is expected to be close to the estimates obtained from Eq. (2.14) by rescaling the Vogt-Wannier value either for ANCA or ANC asymptotics (see the Appendix): $\left.\chi^{j=1}(\kappa)\right|_{\kappa \rightarrow 0} \approx{ }^{\mathrm{ANCA}} \chi_{\mathrm{BW}}^{j=1}$ $=2 \sqrt{1+b^{2} / 3}$ or $\left.\chi^{j=1}(\kappa)\right|_{\kappa \rightarrow 0} \approx \mathrm{ANC}_{\chi_{\mathrm{BW}}^{j=1}}=2 \sqrt{1+5 b^{2} / 18}$. Increasing $\kappa$ from $\kappa=0$ to $\kappa>>1$, the rate coefficients $\chi^{j=1}$ decrease from $\chi_{\mathrm{BW}}^{j=1}$ to $\chi_{\mathrm{L}}=1$ in an undulating manner which is due to the successive opening of the capture channels. For $j=0$, the undulations were analyzed in detail in Ref. 18. For $j=1$, they were discussed in Ref. 6 in connection with the anisotropy of the potential. A useful variable to describe the energy dependence of $\chi$ is the quantity $\sqrt{2 \kappa}$ which, when equal

TABLE IV. Parameters for $\mathrm{H}_{2}+\mathrm{H}_{2}^{+}$collisions.

\begin{tabular}{llll}
\hline \hline$\mu$ & \multicolumn{1}{c}{$Q$} & \multicolumn{1}{c}{$\alpha=\frac{\alpha_{\|}+2 \alpha_{\perp}}{3}$} & $\Delta \alpha=\alpha_{\|}-\alpha_{\perp}$ \\
\hline $1836 m_{e}$ & 0.474 a.u. & 5.437 a.u. & 2.12 a.u. \\
$1.0075 \mathrm{amu}$ & $0.637 \cdot 10^{-26}$ & $0.8057 \AA^{3}$ & $0.314 \AA^{3}$ \\
& $\mathrm{esu} \cdot \mathrm{cm}^{2}$ & $8.057 \cdot 10^{-25} \mathrm{~cm}^{3}$ & $3.14 \cdot 10^{-25} \mathrm{~cm}^{3}$ \\
\hline \hline
\end{tabular}


TABLE V. Scaling coefficients and scaled parameters for $\mathrm{H}_{2}+\mathrm{H}_{2}^{+}$collisions.

\begin{tabular}{lllc}
\hline \hline$R_{\mathrm{L}}=\frac{\sqrt{\mu q^{2} \alpha}}{\hbar}$ & \multicolumn{1}{c}{$E_{\mathrm{L}}=\frac{\hbar^{2}}{\mu R_{\mathrm{L}}^{2}}$} & $a=-\frac{2}{15} \frac{\Delta \alpha}{\alpha}$ & $b=\frac{2}{5} \frac{q Q \mu}{\hbar^{2} R_{L}}$ \\
\hline 100.2 a.u. & $5.450 \cdot 10^{-8}$ a.u. & & \\
$52.9 \AA$ & $1.72 \cdot 10^{-2} \mathrm{~K}$ & -0.052 & 3.48 \\
& $1.20 \cdot 10^{-2} \mathrm{~cm}^{-1}$ & & \\
\hline \hline
\end{tabular}

to $\sqrt{\ell(\ell+1)}=\sqrt{2 \kappa_{\ell}}$, corresponds to the classical opening of the capture channel with the respective $\ell$ in the case of pure charge-induced dipole interaction. The interfragment distance that corresponds to the maximum of the effective potential (the capture distance $\rho_{\ell}$ ) is then equal to $\kappa_{\ell}^{-1 / 2}$; the ratio of the charge-quadrupole interaction to the charge-induced dipole interaction at this distance, $|b| \rho_{\ell}=|b| \kappa_{\ell}^{-1 / 2}$, characterizes the effect of the former in comparison to that of the latter. If, in addition, one ignores the small effect of the anisotropic polarizability, one obtains the data of Table III which describes the qualitative properties of the rate coefficients in different energy regimes and for different values of the charge-quadrupole interaction.

The described approach in the following is used for the numerical calculation of the rate coefficients for capture of $\mathrm{H}_{2}(j=0,1)$ by $\mathrm{H}_{2}{ }^{+}$. The latter event represents an intermediate (and irreversible) stage of the exothermal reaction $\mathrm{H}_{2}+\mathrm{H}_{2}{ }^{+} \rightarrow \mathrm{H}_{3}{ }^{+}+\mathrm{H}$. The lifetime of the intermediate complex $\mathrm{H}_{4}{ }^{+}$is believed to be long enough to lead to statistical behaviour of the complex and its decay into the product channels. Thus the capture rate can be identified with the reaction rate that can be directly measured.

Our previous approximate results for $\mathrm{H}_{2}(j=1)+\mathrm{H}_{2}{ }^{+}$capture (see Fig. 7 of Ref. 7) were presented as scaled temperaturedependent rate coefficients vs. scaled temperatures $k_{\mathrm{B}} T / E_{L}$. Different from this, we now use scaled energy-dependent rate coefficients which appear more appropriate for a detailed analysis of the capture dynamics (see the Appendix) since the

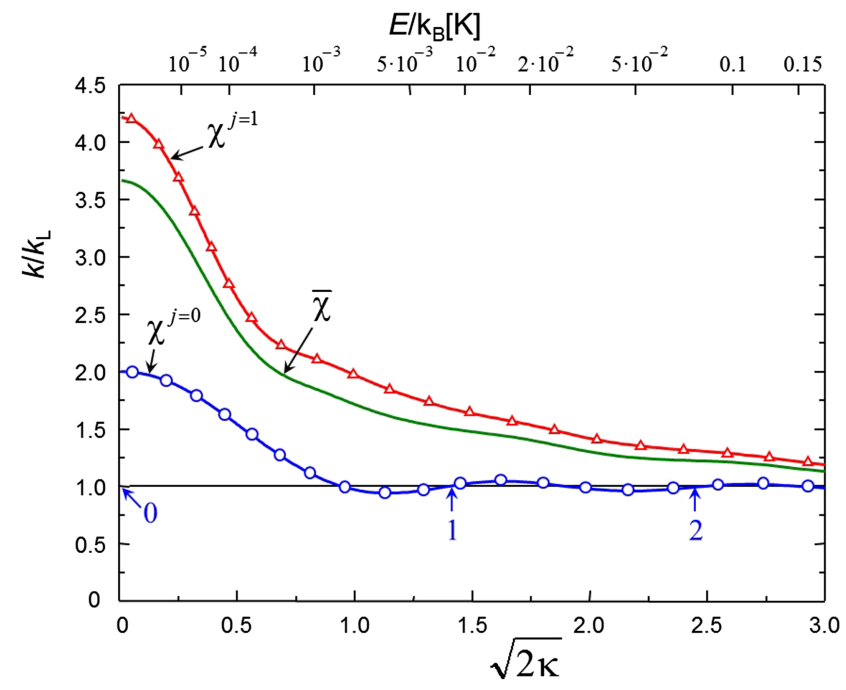

FIG. 2. Scaled rate coefficients for capture of $\mathrm{H}_{2}(j=0,1)$ by $\mathrm{H}_{2}{ }^{+}\left(\chi^{j=0}, \chi^{j=1}\right)$ and mean rate coefficients for a para-ortho mixture $\bar{\chi}=(1 / 4) \chi^{j=0}+(3 / 4) \chi^{j=1}$ (values are given relative to the Langevin limit $k_{L}$; the classical opening of successive channels for a charge-induced dipole potential for $j=0$ is marked by arrows (accurate results from the present work; ANC results are presented in Figs. 4 and 5 in the Appendix).

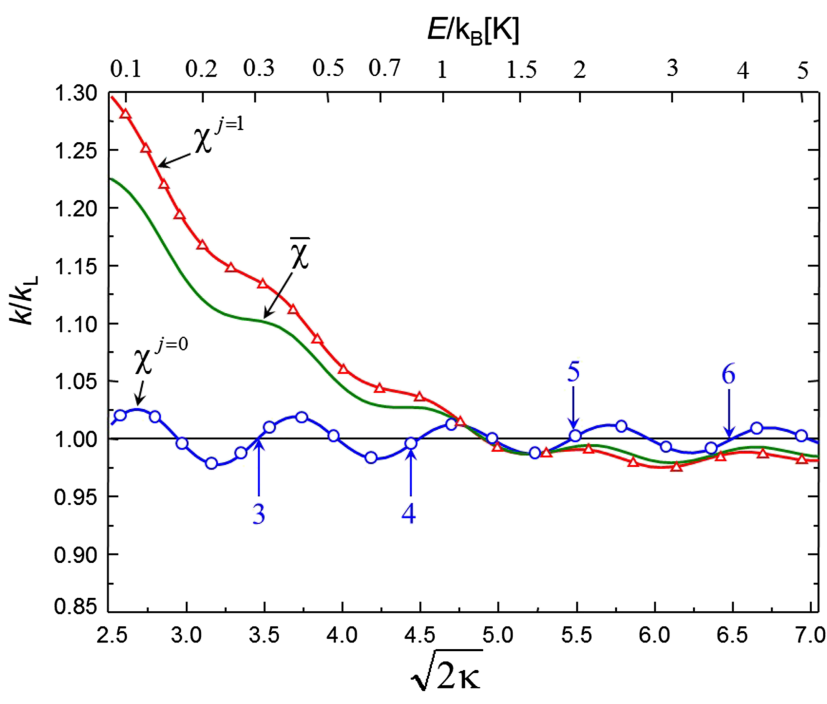

FIG. 3. As Fig. 2, but for larger energies.

structure then is not lost by thermal averaging. Also, energydependent rate coefficients are needed for experiments with well-defined collision energies. The relevant parameters of the calculation are summarized in Tables IV and V.

Figs. 2 and 3 show the resulting $\chi^{j=0}, \chi^{j=1}$, and mean rate coefficients for a para-ortho mixture of $\bar{\chi}=(1 / 4) \chi^{j=0}$ $+(3 / 4) \chi^{j=1}$.

The scale of the lower horizontal axis is chosen according to the condition that the undulations of $\chi^{j=0}$ are evenly spaced. The upper horizontal axis corresponds to collision energies in K. Compared to Fig. 2, the vertical axis in Fig. 3 is expanded in order to show more details close to the Langevin limit. One notices a smaller amplitude of the undulations for $\chi^{j=1}$ and $\bar{\chi}$ than for $\chi^{j=0}$ which is due to a more efficient quenching of the interferences for $j=1$ by the larger number of contributing channels. The general pattern of the energy dependence of the rate coefficients can be understood in terms of the above discussion such as summarized in Table III. More details can be learned from an inspection of the decoupling ANC approximations (such as given in the Appendix). Here we only mention that the decoupling ANC approximation yields a total rate coefficient ${ }^{\mathrm{ANC}} \chi^{j=1}$ that is very close to its accurate counterpart $\chi^{j=1}$ such as determined here and illustrated in Figs. 2 and 3. We also find that the classical ANC rate coefficient (ACNCl), ${ }^{\mathrm{ANCCl}} \chi^{j=1}$, well approximates ${ }^{\mathrm{ANC}} \chi^{j=1}$ for collision energies above $5 \cdot 10^{-2} \mathrm{~K}$. At this energy, $\chi^{j=1}$ is about $40 \%$ higher than $\chi^{j=0}$.

\section{v. CONCLUSIONS}

The capture of a quadrupolar diatom by an ion, during the course of the collision, is accompanied by a change of the rotational polarization of the molecule with respect to the collision axis. The classical explanation of this effect corresponds to the precession and nutation of the intrinsic angular momentum and its coupling to the radial motion of collision partners. The quantum counterpart of this behavior is expressed by a set of coupled radial wave equations. For low collision energies, these equations can be partially decoupled within the 
perturbed rotor approximation which assumes that the intrinsic angular momentum $j$ is a good quantum number. Within this approximation, we have calculated rate coefficients for capture of $\mathrm{H}_{2}(\mathrm{j}=0,1)$ by $\mathrm{H}_{2}{ }^{+}$from ultra-low energies (few classically open channels) up to energies where many channels are classically open and the capture rate coefficients converge to their Langevin limit. We also analyzed the axially nonadiabatic channel approximation (ANC) which diagonalizes the Coriolis interaction at fixed radial coordinates and ignores the radial nonadiabatic coupling between ANC channels. The comparison with the accurate results shows a very good performance of the ANC approximation, from the classical down to the Bethe-Wigner limit. In the latter range, we found a non-negligible effect of the diagonal non-adiabatic correction incorporated into the ANC potential. The main reason for the adequacy of the ANC approximation is the correct description of the relocking of the intrinsic angular momentum of $\mathrm{H}_{2}$, which significantly affects the ANC potentials. In this respect, the standard AC approximation fails badly at ultra-low energies. Rather unexpectedly, we have found that the classical ANC approximation well reproduces the quantal results already at energies when only 5 or 6 capture channels are classically open, i.e., at an energy corresponding to $0.1 \mathrm{~K}$. At this energy, the capture rate coefficient for $j=1$ is about $25 \%$ higher than that for $j=0$. For a standard ortho-para mixture of $\mathrm{H}_{2}$, the mean capture rate coefficient at $0.1 \mathrm{~K}$ is expected to be about $20 \%$ higher than that for para-hydrogen.

We close by mentioning that experimental studies of the reaction $\mathrm{H}_{2}+\mathrm{H}_{2}{ }^{+} \rightarrow \mathrm{H}+\mathrm{H}_{3}{ }^{+}$are being conducted now down to temperatures such as considered in the present work. ${ }^{19}$ The lowest temperature attained so far is about $300 \mathrm{mK} .{ }^{19}$ At this temperature, the rate coefficient for $j=0$ is dominated by four channels, $\ell=0,1,2,3$ (see Figs. 2 and 3, blue line). For $j=1$ it is dominated by eight decoupled channels $J_{s}=1_{-1}, 1_{0}$, $2_{-1}, 2_{0}, 3_{-1}, 3_{0}, 4_{-1}, 4_{0}$ (arrows in Figs. 4 and 5), with four channels $J_{s}=1_{-1}, 2_{-1}, 3_{-1}, 4_{-1}$ from the $s=-1$ manifold and

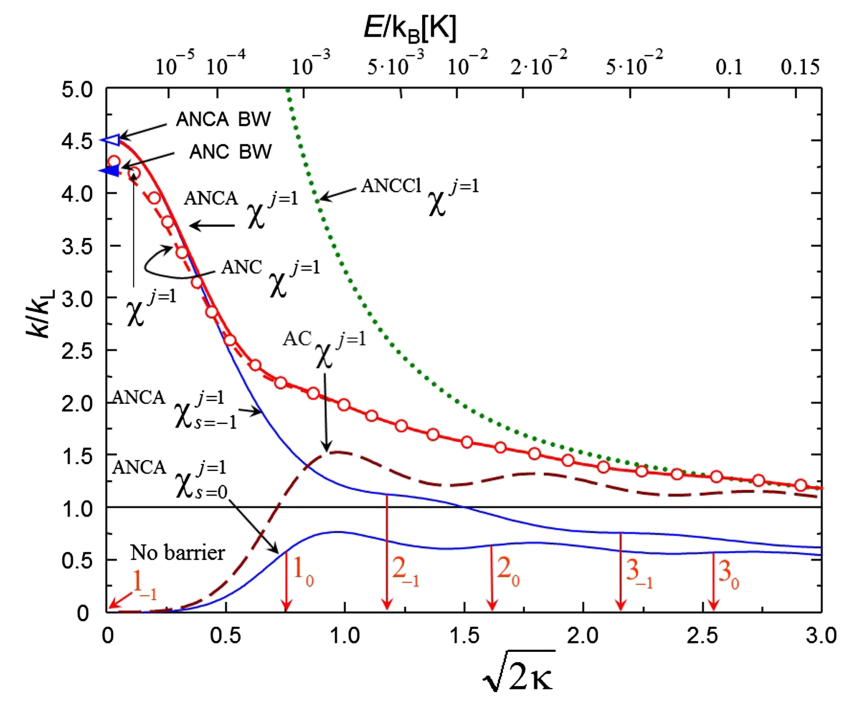

FIG. 4. Total and partial scaled rate coefficients for capture of $\mathrm{H}_{2}(j=1)$ by $\mathrm{H}_{2}{ }^{+}$in different approximations across the ultra-low (two classically open channels) and very-low (several classically open channels) energy range. (The classical opening of successive channels for ANC potentials is marked by $J_{S}$ symbols and arrows. BW = Bethe-Wigner limits.)

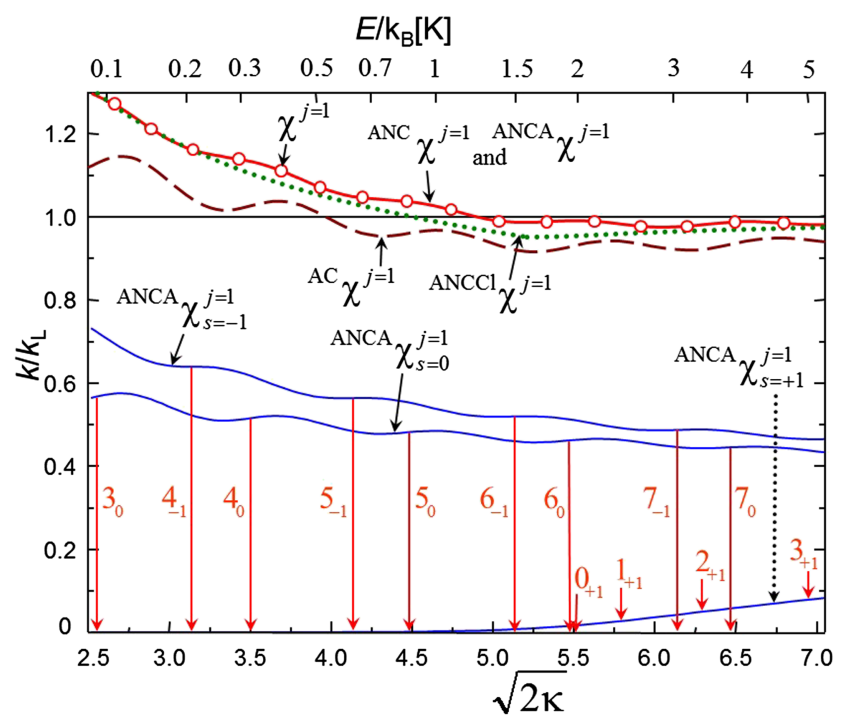

FIG. 5. As Fig. 4, but for larger energies.

four channels $J_{s}=1_{0}, 2_{0}, 3_{0}, 4_{0}$ from the $s=0$ manifold. These two manifolds are contributing almost equally (two blue lines in Fig. 4 and two upper blue lines in Fig. 5). All channels $J_{+1}$ from $s=1$ manifold are closed for the capture at this temperature and their contribution becomes noticeable only above $5 \mathrm{~K}$ (the lowest blue line in Fig. 5). The agreement between the experiment and theory for the mean rate coefficients for a para-ortho mixture of $\mathrm{H}_{2}$ is then within experimental error.

\section{ACKNOWLEDGMENTS}

We thank Professor F. Merkt for communicating his experimental results ${ }^{10,19}$ to us.

\section{APPENDIX: CAPTURE DYNAMICS FOR $\mathrm{H}_{2}(j=1)+\mathrm{H}_{2}{ }^{+}$ IN THE DECOUPLING ANC APPROXIMATION}

In a decoupling approximation, the sum in Eq. (4.1) is simplified to a single term. Since then $\Gamma$ is redundant, we write

$$
\left.\Psi_{\gamma ; \Gamma}^{J j}(\rho, \Xi)\right|_{\Gamma=\gamma} \equiv \Psi_{\gamma}^{J j}(\rho, \Xi)=\chi_{\gamma}^{J j}(\rho)|J, j, \gamma ; \rho, \Xi\rangle,
$$

with $\chi_{\gamma ; \Gamma}^{J j}(\rho)$ following from the wave equation

$$
\eta_{\gamma \gamma}^{J j} \chi_{\gamma}^{J j}=E \chi_{\gamma}^{J j}
$$

In the application to the decoupling ANC approximation discussed below, we put $\gamma=s$ and consider the diagonal elements of the Hamiltonian matrices ${ }^{A N C} v_{s s}^{J, j=1}$ in Eqs. (2.11) and (2.12) as independent potentials that lead to capture. The capture in the field of these potentials is characterized by the probabilities ${ }^{\mathrm{ANC}} P_{s}^{J, j=1}$. The latter define partial and total rate coefficients as

$$
\begin{aligned}
{ }^{\mathrm{ANC}} \chi^{j=1} & =\sum_{s} \mathrm{ANC}_{\chi_{s}^{j=1}}, \\
{ }^{\mathrm{ANC}} \chi_{s}^{j=1} & =\frac{1}{6 \kappa} \sum_{J}(2 J+1)^{\mathrm{ANC}} P_{s}^{J, j=1} .
\end{aligned}
$$

In the following, we use ${ }^{\mathrm{ANC}} P_{s}^{J, j=1}$ for the definition of characteristic energies and threshold values $J_{S}$ for capture in individual channels, as well as for the calculation of ${ }^{{ }^{A N C}} \chi_{s}^{j=1}$ and 
${ }^{\mathrm{ANC}} \chi^{j=1}$ in order to interpret numerically calculated $\chi^{j=1}$ (for simplicity, later we suppress the superscript ANC).

We define the characteristic energies $\varepsilon_{J, s}^{j=1}$ as heights of the barriers for $J \neq 1, s \neq-1$ and put $\varepsilon_{J, s}^{j=1}=0$ for $J=1$, $s=-1$. In the former case, the capture probabilities $\left.P_{s}^{J, j=1}(\varepsilon)\right|_{\varepsilon=\varepsilon_{J, s}^{j=1}}$ are approximately equal to $1 / 2$, while for the latter case the probability vanishes but the partial rate coefficient equals its BW limit, ${ }^{\mathrm{ANC}} \chi_{\mathrm{BW}}^{j=1}=2 \sqrt{1+5 b^{2} / 18}$.

The values of $\sqrt{2 \kappa_{J, s}^{j=1}}$ that correspond to $\varepsilon_{J, s}^{j=1}$ are shown on the horizontal axis in Fig. 4 (non-WKB energy range) and Fig. 5 (WKB energy range) by vertical arrows labeled by $J_{s}$. This is similar to Figs. 2 and 3. Also shown are the partial decoupled rate coefficients ${ }^{\mathrm{ANC}} \chi_{s}^{j=1}$, the total rate coefficients ${ }^{\mathrm{ANCA}} \chi^{j=1}$ in the ANCA approach, and the classical rate coefficients ${ }^{\mathrm{ANCCl}} \chi^{j=1}$.

The following features deserve mentioning:

(1) The ANC results are very close to the accurate results. This implies that the radial nonadiabatic coupling between ANC channels is nearly negligible.

(2) The influence of the radial nonadiabatic correction on the ANC potentials is demonstrated by a comparison of the accurate and the ANC and ANCA rate coefficients. The difference between ANC and ANCA rate coefficients, which is due to the diagonal nonadiabatic correction, is discernable only at ultra-low energies where the rate is determined by the capture in the first channel $J=1, s=-1$.

(3) The result of the discrete nature of the quantum angular momentum is demonstrated by a comparison of the classical $\mathrm{ANCCl}$ and the quantum ANC rate coefficients. The divergence of ${ }^{{ }^{A N C C l}} \chi$ in the limit of low energies arises from the continuous distribution of the total angular momentum. The latter approximation becomes acceptable for energies at which channels with $J>3$ classically open (with quantum/classical deviations less than $10 \%$ for energies above $5 \cdot 10^{-2} \mathrm{~K}$ ).

(4) The result of relocking is demonstrated by the sequence of the alignment indices at the opening of channels. This sequence can be read from the ordinate of the labeled points in Fig. 1 corresponding to specific values of $J, s$.

(5) The effect of nutations is demonstrated by a comparison of ANC and AC rate coefficients. We see that the AC approximation (where nutation is ignored) fails at low energies (below $5 \cdot 10^{-2} \mathrm{~K}$ ). At higher energies, the quantum $\mathrm{AC}$ approximation performs even worse than the classical ANC approximation.

(6) The partial contributions ${ }^{\mathrm{ANCA}} \chi_{s=-1}^{j=1},{ }^{\mathrm{ANCA}} \chi_{s=0}^{j=1}$, and ${ }^{A N C A} \chi_{s=1}^{j=1}$ follow the expected relation ${ }^{A N C A} \chi_{s=-1}^{j=1}$ $>{ }^{\mathrm{ANCA}} \chi_{s=-0}^{j=1}>{ }^{\mathrm{ANCA}} \chi_{s=+1}^{j=1}$. The late appearance of the contribution ${ }^{\mathrm{ANCA}} \chi_{s=+1}^{j=1}$ is responsible for the drop of the total rate ${ }^{\mathrm{ANCA}} \chi^{j=1}$ below the Langevin limit before this is finally reached after passing through a shallow minimum.

(7) The late appearance of the contribution ${ }^{\mathrm{ANCA}} \chi_{s=+1}^{j=1}$, arising from the asymptotically strongly repulsive ANC potential, suggests that the state $\Omega=0$ in the k-helicity basis also does not contribute substantially to $\chi^{j=1}$ at low energies. Then writing $\chi^{j=1}=(1 / 3) \sum_{\Omega= \pm 1} \chi_{\Omega}^{j=1}$ and taking into account the relation $\chi_{\Omega=1}^{j=1}=\chi_{\Omega=-1}^{j=1}$, we get a capture rate coefficient for the $\mathrm{k}$-helicity rotationally polarized rotor of $\chi_{\Omega=1}^{j=1}=(3 / 2) \chi^{j=1}$.

(8) The relation between different partial rate coefficients follows the predictions from the corresponding expressions for AC and ANC potentials

$$
\begin{aligned}
& { }^{\mathrm{AC}} \chi_{1}^{j=1}={ }^{\mathrm{AC}} \chi_{-1}^{j=1}>{ }^{\mathrm{AC}} \chi_{0}^{j=1}, \\
& { }^{\mathrm{ANC}} \chi_{-1}^{j=1}>{ }^{\mathrm{ANC}} \chi_{0}^{j=1}>{ }^{\mathrm{ANC}} \chi_{1}^{j=1}, \\
& { }^{\mathrm{ANC}} \chi_{-1}^{j=1}>{ }^{\mathrm{AC}} \chi_{-1}^{j=1}={ }^{\mathrm{AC}} \chi_{1}^{j=1}, \\
& { }^{\mathrm{ANC}} \chi_{0}^{j=1}={ }^{\mathrm{AC}} \chi_{-1}^{j=1}={ }^{\mathrm{AC}} \chi_{1}^{j=1}, \\
& { }^{\mathrm{ANC}} \chi_{1}^{j=1}<{ }^{\mathrm{AC}} \chi_{0}^{j=1} .
\end{aligned}
$$

In the limit $\varepsilon \rightarrow 0$, all rate coefficients ${ }^{\mathrm{AC}} \chi_{\omega}^{j=1}$ as well as ${ }^{\mathrm{ANC}} \chi_{s=0}^{j=1},{ }^{\mathrm{ANC}} \chi_{s=1}^{j=1}$ disappear. The remaining partial ANC contribution ${ }^{\mathrm{ANC}} \chi_{-1}^{j=1}$ is expressed through the respective BetheWigner radius as

$$
\begin{aligned}
\lim _{\varepsilon \rightarrow 0}{ }^{\mathrm{ANC}} \chi^{j=1}(\varepsilon) & =\lim _{\varepsilon \rightarrow 0}{ }^{\mathrm{ANC}} \chi_{-1}^{j=1}(\varepsilon)={ }^{\mathrm{ANC}} \rho_{B W}^{j=1} \\
& =2 \sqrt{1+5 b^{2} / 18}=4.18 .
\end{aligned}
$$

The corresponding expression for $j=0$ reads $^{18}$

$$
\lim _{\varepsilon \rightarrow 0} \chi^{j=0}(\varepsilon)=\rho_{B W}^{j=0}=2 .
$$

It appears also of interest that, at the energy when $\bar{\chi}$ noticeably exceeds its Langevin limit (e.g., by $20 \%$ at $0.1 \mathrm{~K}$, see Fig. 4), the classical ANC approach provides a reasonable approximation.

The decoupling approximation allows one to estimate possible effects of additional interactions between $\mathrm{H}_{2}$ and $\mathrm{H}_{2}{ }^{+}$ neglected in the present treatment: the quadrupole-quadrupole (qq) interaction and the molecular orbital (MO) overlap (Franck-Condon, FC) interaction responsible for the symmetric charge transfer. The relative correction of the qq interaction to the cq interaction is in the order of $Q / e R^{2} \approx r_{e}^{2} / R^{2}$ where $r_{e}^{2}$ is the equilibrium distance between the protons in $\mathrm{H}_{2}$ or $\mathrm{H}_{2}{ }^{+}$. This correction makes a negligible contribution to the capture in all channels that become open below the energy $\varepsilon_{J, s}^{j=1}$. For the channel $J_{s}$, the relative qq correction amounts to about $r_{e}^{2} / R_{J, s}^{2}$. This ratio, for $r_{e}^{2}$ of about 1 a.u., reads $r_{e}^{2} / R_{J, s}^{2} \approx 1 / R_{L}^{2}$ (a.u.) $\rho_{J, s}^{2}$. With $R_{L}$ (a.u.) $\approx 10^{2}$ (see Tables IV and $\mathrm{V}$ ), the relative correction of the qq interaction to the cq interaction at the characteristic capture distance $\rho_{J, s}$ amounts to $10^{-4} / \rho_{J, s}^{2}$. Reading the values of $\rho_{J, s}$ from Fig. 1 (symbols on the lines), we find that this correction is negligible for all the channels shown in this figure $(J \leq 10)$ and while referring to Fig. 5, we see that qq interaction can be certainly neglected for collision energies $E / k_{\mathrm{B}}<5 \mathrm{~K}$. An estimation of the MOFC interaction, by referring to the asymptotic theory of the exchange interaction, ${ }^{20}$ indicates that the Franck-Condon overlap of MO on two centers (that could lead to the symmetric 
charge transfer) does not show up in the capture dynamics. We also mention that there are no other nuclear spin restrictions in the capture beside those related to the mean rate coefficient for the equilibrium para-ortho mixture of $\mathrm{H}_{2}$ (see Figs. 2 and 3).

Summarizing, we note again the very good performance of the uncoupled ANC approximation across the low-temperature range $\left(T<<B h c / k_{B}\right)$. At least this applies to the $\mathrm{H}_{2}(j=1)$ $+\mathrm{H}_{2}{ }^{+}$capture. Deficiencies of the uncoupled ANC approximation may show up at higher energies, but this would not change the rate coefficient since the latter is already close to its Langevin counterpart.

${ }^{1}$ E. I. Dashevskaya, I. Litvin, and E. E. Nikitin, J. Phys. Chem. A 110, 2876 (2006).

${ }^{2}$ E. I. Dashevskaya, I. Litvin, E. E. Nikitin, and J. Troe, Mol. Phys. 108, 873 (2010).

${ }^{3} \mathrm{~J}$. Troe, in State Selected and State to State Ion Molecule Reaction Dynamics, Part 2: Theory, Advances in Chemical Physics, edited by M. Baer and C. Y. Ng (Wiley, New York, 1992), Vol. 82, pp. 485.

${ }^{4} \mathrm{~N}$. Andersen and K. Bartschat, Polarization, Alignment, and Orientation in Atomic Collisions (Springer, New York, 2001).
${ }^{5}$ E. I. Dashevskaya, I. Litvin, E. E. Nikitin, and J. Troe, J. Chem. Phys. 120, 9989 (2004).

${ }^{6}$ E. I. Dashevskaya, I. Litvin, E. E. Nikitin, I. Oref, and J. Troe, J. Phys. Chem. A 108, 8703 (2004).

${ }^{7}$ E. I. Dashevskaya, I. Litvin, E. E. Nikitin, and J. Troe, J. Chem. Phys. 122, 184311 (2005).

${ }^{8}$ Y. Shagam, A. Klein, W. Skomorowski, R. Yun, V. Averbukh, Ch. P. Koch, and E. Narevicius, Nat. Chem. 7, 921 (2015).

${ }^{9}$ M. Lara, P. G. Jambrina, F. J. Aoiz, and J.-M. Launay, J. Chem. Phys. 143, 204305 (2015).

${ }^{10}$ P. Allmendinger, J. Deiglmayr, O. Schullian, K. Hoeveler, J. A. Agner, H. Schmutz, and F. Merkt, ChemPhysChem 17, 3596 (2016).

${ }^{11}$ E. E. Nikitin and R. N. Zare, Mol. Phys. 82, 85 (1994).

${ }^{12}$ D. A. Case and D. R. Herschbach, Mol. Phys. 30, 1537 (1975).

${ }^{13}$ D. A. Case and D. R. Herschbach, J. Chem. Phys. 64, 4212 (1976).

${ }^{14}$ E. I. Dashevskaya, E. E. Nikitin, and J. Troe, J. Chem. Phys. 93, 7803 (1990).

${ }^{15}$ H. Bethe, Phys. Rev. 47, 747 (1935).

${ }^{16}$ E. Wigner, Phys. Rev. 73, 1002 (1948).

${ }^{17}$ E. Vogt and G. H. Wannier, Phys. Rev. 95, 1190 (1954).

${ }^{18}$ E. I. Dashevskaya, I. Litvin, A. I. Maergoiz, E. E. Nikitin, and J. Troe, J. Chem. Phys. 118, 7313 (2003).

${ }^{19}$ P. Allmendinger, J. Deiglmayr, K. Hoeveler, O. Schullian, and F. Merkt, J. Chem. Phys. 145, 244316 (2016).

${ }^{20}$ E. E. Nikitin and S. Ya. Umanskii, Theory of Slow Atomic Collisions (Springer-Verlag, Berlin, Heidelberg, 1984). 